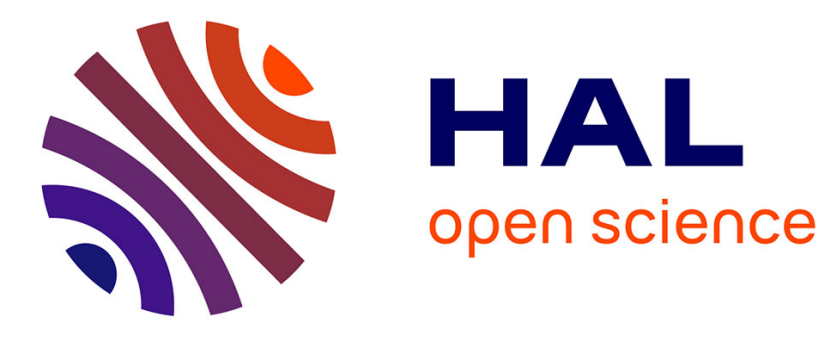

\title{
Spatio-temporal registration of embryo images
}

Léo Guignard, Christophe Godin, Ulla-Maj Fiuza, Lars Hufnagel, Patrick

Lemaire, Grégoire Malandain

\section{To cite this version:}

Léo Guignard, Christophe Godin, Ulla-Maj Fiuza, Lars Hufnagel, Patrick Lemaire, et al.. Spatiotemporal registration of embryo images. ISBI - International Symposium on Biomedical Imaging, Apr 2014, Pekin, China. hal-00919142

\section{HAL Id: hal-00919142 \\ https://hal.inria.fr/hal-00919142}

Submitted on 16 Dec 2013

HAL is a multi-disciplinary open access archive for the deposit and dissemination of scientific research documents, whether they are published or not. The documents may come from teaching and research institutions in France or abroad, or from public or private research centers.
L'archive ouverte pluridisciplinaire HAL, est destinée au dépôt et à la diffusion de documents scientifiques de niveau recherche, publiés ou non, émanant des établissements d'enseignement et de recherche français ou étrangers, des laboratoires publics ou privés. 


\title{
SPATIO-TEMPORAL REGISTRATION OF EMBRYO IMAGES
}

\author{
L. Guignard ${ }^{\star \dagger}$ C. Godin ${ }^{\dagger}$ U.-M. Fiuza ${ }^{\star}$ L. Hufnagel ${ }^{\ddagger}$ P. Lemaire Le Malandain $^{\S}$ \\ ${ }^{\star}$ CRBM, UMR 5237, CNRS, Univ. Montpellier 1 \& 2, 34293 Cedex 5 Montpellier, France \\ ${ }^{\dagger}$ Inria, Virtual Plants team, UMR AGAP, 34095 Montpellier, France \\ $\S$ Inria, Morpheme team, 06900 Sophia Antipolis, France \\ ${ }^{\ddagger}$ EMBL, Cell Biology and Biophysics Unit, Heidelberg, Germany.
}

\begin{abstract}
Current imaging techniques can capture temporal sequences of 3D images with very high time resolution over several hours. Comparing sequences covering the same time period opens the way to the study of developmental variability. Stitching together sequences captured from different embryos may help producing a sequence covering the whole development of the animal of interest. For this, it is necessary to align two sequences in both time and space.

We present here a method to align two $3 \mathrm{D}+\mathrm{t}$ time series, based on the detection and pairing of 3D+t landmarks. These landmarks, which correspond to periods of fast morphogenetic change, are deduced from the analysis of the non-linear transformations that allow to co-register pairs of consecutive 3D images in each sequence.
\end{abstract}

Index Terms - Image registration, Image sequence processing, Fluorescence microscopy, Light sheet microscopy, Embryogenesis, Morphogenesis

\section{INTRODUCTION}

Animal and plant morphogenesis is a highly dynamic process spanning several temporal and spatial scales. One challenge is to describe cell and/or embryo shapes through development, to analyze their dynamics and variability within and between species and to correlate this information with gene activity $[1,2]$. For this, it is necessary to compare the development of several embryos in order to identify similarities and variations in morphogenetic events.

Recent progress in light microscopy allows the capture of 3D images of entire live embryos at a sub-cellular level of resolution with a high rate of acquisition and without interfering with development [3]. These protocols produce $3 \mathrm{D}+\mathrm{t}$ images depicting the development. Comparing or fusing such sequences captured from different embryos is a challenging task, but of crucial importance. While it is generally not possible to image an embryo throughout its whole development, one can generate developmental sequences from different individuals, with partial temporal overlap. Stitching together such time series can be used to produce a consensual representation of the whole development, and would also allow to identify variations in these programs.

There are however two main challenges. Firstly, it is experimentally difficult or impossible to orient the embryos in a standardized manner during imaging. Moreover embryos often progressively drift in the field of view during long time-lapse sessions. It is thus necessary to realign spatially each sequence so that the embryos are in the same orientation, and thus can be easily compared. Secondly, one needs to temporally align these time series over their periods of overlap. Because of the very large size of such datasets (up to several hundred 3D images per sequence), alignment methods have to be as automated as possible.

We propose here a landmark-based registration method for two $3 \mathrm{D}+\mathrm{t}$ time series. These landmarks are extracted from the analysis of the sequences. More precisely, in a given time series, every pair of consecutive 3D images are co-registered with a non-linear transformation, and the largest regional deformations are extracted from the whole sequences, yielding 3D+t landmarks. Alignment of two independent sequences is then achieved by finding the optimal pairings between two sets of 3D+t landmarks.

\section{DATA DESCRIPTION}

We used the simple marine invertebrate chordate Phallusia mammillata (P.m.) to test and develop our algorithms. Embryonic development of this species is highly similar across individuals, because the timing and orientation of cell divisions are highly stereotyped. As a consequence all cells can be unambiguously named and recognized up to the 112-cell stage, when the first major morphogenetic process, gastrulation is initiated.

We imaged live embryos using a lightsheet microscope $(\mathrm{Mu}-$ ViSPIM, EMBL, Heidelberg [4]). To image membranes, embryos were soaked in a lipophilic dye (FM4-64). The dye was excited with a 594nm laser (Cobolt $\mathrm{AB}$ ) and the emitted light was collected by two opposing $25 \mathrm{x}$ Nikon water dipping objective lens (NA 1.1) and after passing thought a long-pass filter (BLP01-594R-25, Semrock), was collected with a Hamamatsu Flash 4 SCMOS camera. At each time point, two perpendicular 3D image stacks, each with $1200 \times 1200$ pixels per plane and a total of 200 planes were acquired by both cameras. This resulted in a total of four views ( 0 , $90,180,270$ degrees) of the specimen each with a lateral resolution of $0.26 \mu \mathrm{m} \times 0.26 \mu \mathrm{m}$ and $1 \mu \mathrm{m}$ plane spacing. This procedure was repeated every two minutes. To obtain an $3 \mathrm{D}$ image with isotropic resolution, the 4 image stacks were fused into a single 3D dataset as described in [5].

For the purpose of this paper we will use time-series from two different embryos, each counting 50 time points (1h40 min). Both time series start at the 32-cell stage and end after the 112-cell stage. The imaging temperatures were different $\left(20^{\circ} \mathrm{C}\right.$ and $\left.18^{\circ} \mathrm{C}\right)$ so that both embryos exhibit slightly different developmental kinetics. Fig. 1 depicts 2D sections of some 3D images at different timepoints extracted from one sequence as well as the corresponding 3D renderings. 


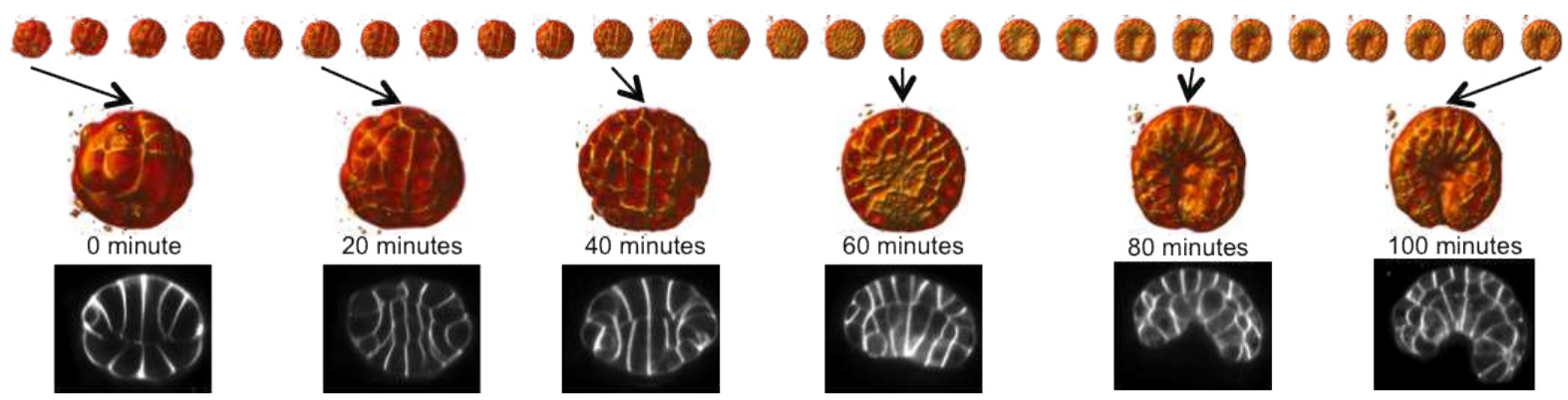

Fig. 1. Phallusia mammillata embryo from 32 cells stage to 112 cells stage, 100 minutes later. The first row shows the $3 \mathrm{D}$ renderings of the voxel images (Amira) every 4 minutes, while the other rows display respectively some magnified views and corresponding cross-sections.

\section{INTRA-SEQUENCE REGISTRATION}

\subsection{Registration method}

Registration is a frequently addressed issue in the literature [6, 7]. Although the choice of a particular registration method may not be critical, it is important that the chosen method would be robust with respect to topological changes. For that reason, we chose to use a block matching scheme [8] to compute either linear or non-linear transformations. Such a scheme is comparable to the ICP method [9] except that iconic primitives are matched instead of points.

Registration aims at the computation of the transformation $T_{F \leftarrow R}$ that will allow to resample a floating image $I_{F}$ onto a reference image $I_{R}$. The transformation $T_{F \leftarrow R}$ is iteratively computed by integrating incremental transformations $\delta T^{i}$, ie $T_{F \leftarrow R}^{i+1}=$ $\delta T^{i} \circ T_{F \leftarrow R}^{i}$.

At iteration $i$, blocks $b_{R}$ of the reference image $I_{R}$ are compared to blocks $b_{F}$ of the floating image $I_{F}$, the best block pairing (the one that yields the best iconic measure, here the normalized correlation) yield a point pairing, $\left(c_{R}, c_{F}\right)$, by associating the block centers. The incremental transformation is then estimated by

$$
\delta T^{i}=\arg \min _{\delta T} \sum\left\|c_{F}-\delta T \circ T_{F \leftarrow R}^{i} c_{R}\right\|^{2}
$$

Linear transformations are computed with a Least Trimmed Squares [10] method that allows to discard outlier pairings.

The non-linear transformation is represented by a dense vector field. The transformation update follows then the following steps: 1. the sparse pairing field $\left(c_{R}, c_{F}\right)$ is transformed into a dense field by Gaussian interpolation (this Gaussian interpolation also acts as a fluid regularization); 2 . outlier pairings are discarded; 3 . the remaining pairings are transformed into a dense field by Gaussian interpolation; 4. this dense field is composed with the transformation found at the previous iteration; 5 . the resulting transformation is smoothed by a second Gaussian filter that acts as an elastic regularization.

\subsection{Pairwise registration}

Consider a temporal sequence of 3D images, $\left\{I_{t}\right\}$ for $t=1 \ldots N$. We aim at co-registering a pair of images captured at two different time points (i.e. $I_{t}$ and $I_{t+\delta t}$ ). Since a $\delta t$ of 2 minutes does not capture deformation of sufficient magnitudes we choose $\delta t=4$ minutes. We want to be sure to capture sufficient deformations to identify cell division events.

We first co-register every pair of successive images of the time series with a rigid transformation : 3D translation + rotation representing (and then allowing to compensate for) the small displace- ments of the embryo that may occur during the acquisition. Hence, we get a series of rigid transformations $R_{t \leftarrow t+\delta t}$ allowing to resample $I_{t}$ onto $I_{t+\delta t}$.

To compensate for the displacements along the whole sequence, a reference image is chosen (here $I_{N}$ ), and all the transformations $R_{t \leftarrow N}$ are calculated by transformation composition, which allows to resample all $I_{t}$ images into $I_{N}$ frame

$$
I_{N \leftarrow t}=I_{t} \circ R_{t \leftarrow N}=I_{t} \circ R_{t \leftarrow t+\delta t} \circ \ldots \circ R_{N-\delta t \leftarrow N}
$$

From now on, it is assumed that the sequence is compensated for the small rigid displacements (ie $I_{N \leftarrow t}$ is now denoted $I_{t}$ ).

The remaining deformations that exist between two successive images can be captured thanks to a non-linear registration step. We then compute the non-linear transformations $T_{t+\delta t \leftarrow t}$ thereby resampling $I_{t+\delta t}$ in the same frame as $I_{t}$.

The non-linear transformation $T$ is encoded by a vector field $\mathbf{v}$ such that $T(m)=m+\mathbf{v}(m)$, the modulus of the vector $\mathbf{v}(m)$ indicating the local amount of variation at point $m$. Fig. 2 depicts such a vector field calculated from the registration of $3 \mathrm{D}$ images at two successive time-points: it demonstrates that the deformations are regionally localized, suggesting that these areas are of interest from a morphogenesis point of view.

\subsection{Registration assessment}

To assess the registration quality, we used a distance between the two images based on a comparison of membranes location. The distance is small when the membranes are mainly overlaping. For this, we take advantage of the dye that makes the membranes hyper-intense and extract them by thresholding. More precisely, $10 \%$ on average of the image represents membranes, so the membrane set $\mathcal{M}(I)$ of image $I$ is defined by the binary image resulting of the thresholding of $I$ by the intensity representing the $90^{\text {th }}$ percentile of the cumulative histogram. Although not perfect, such a membrane definition is sufficient for a rough registration assessment.

Let $I_{F}$ and $I_{R}$ be respectively a floating and a reference image, $T_{F \leftarrow R}$ the non-linear transformation from $I_{R}$ to $I_{F}, I_{F \rightarrow R}$ the floating image resampled onto the reference one $\left(I_{F \rightarrow R}=I_{F} \circ T_{F \leftarrow R}\right)$, and $\mathcal{M}_{R}$ and $\mathcal{M}_{F \rightarrow R}$ the membrane sets of respectively $I_{R}$ and $I_{F \rightarrow R}$. We define a registration score $S$ by measuring the average distance from one membrane set to the other thanks to

$$
S\left(I_{F \rightarrow R}, I_{R}\right)=\frac{1}{\left|\mathcal{M}_{R}\right|} \sum_{m \in \mathcal{M}_{R}} \min _{m^{\prime} \in \mathcal{M}_{F \rightarrow R}}\left\|m m^{\prime}\right\|
$$

Fig. 3 shows the evolution of this score from rigid registration, ie $S\left(I_{t+\delta t}, I_{t}\right)$, where the small rigid displacements have already been compensated for, to non-linear registration, ie $S\left(I_{t+\delta t \rightarrow t}, I_{t}\right)$. 

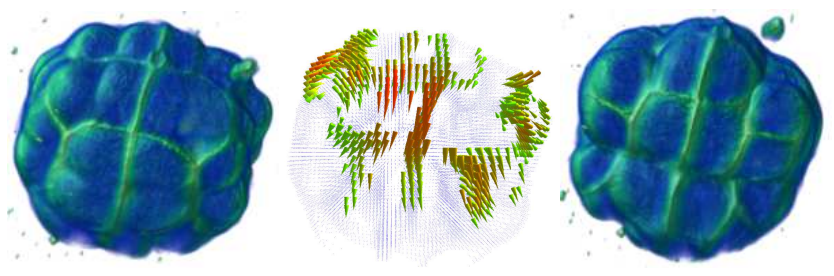

Fig. 2. A cellular division occurs between 16 minutes (rendering at left) and 20 minutes (rendering at right (Amira)) for the embryo of Fig. 1. The non-linear registration that co-registers both images exhibits some high deformations, represented by large vectors (at the middle; only vector with a norm greater than $7.5 \mu \mathrm{m}$ are displayed).

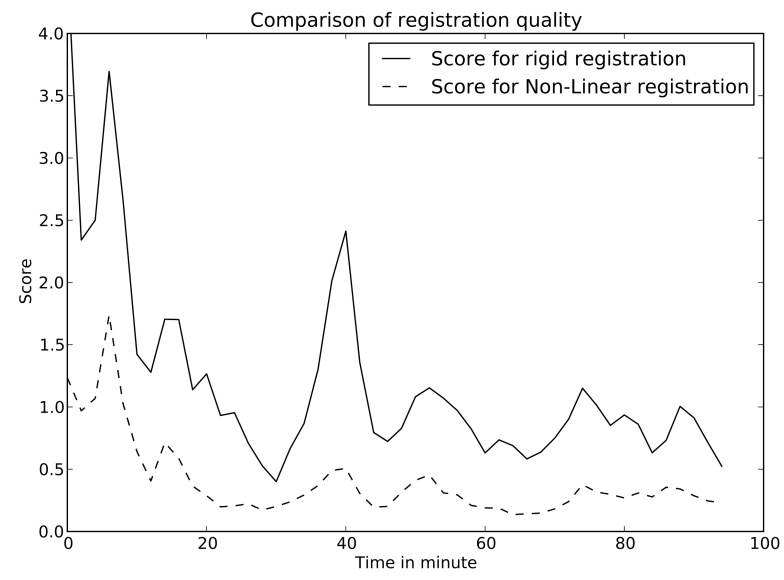

Fig. 3. Registration score over time for both rigid and non-linear registration.

\section{ALIGNEMENT OF INDEPENDENT 3D+T TIME SERIES}

\subsection{Definition of 3D+t landmarks}

The development of the embryo is punctuated by regional deformations at precise steps. For example, during the cell division process the dividing cells round up, which causes local deformation. Also, during gastrulation part of the embryo invaginates, a process that creates regional deformations. We use these spatially and temporally localized events as $3 \mathrm{D}+\mathrm{t}$ landmarks to guide the co-registration of two morphogenesis sequences.

We then propose to use the deformations extrema as $3 \mathrm{D}+\mathrm{t}$ landmarks. Let us denote the set of landmarks to be built by $\mathcal{L}=\left\{L_{i}\right\}$. A 3D+t landmark $L_{i}$ is defined by a time $t_{i}$ and a spatial position $x_{i}$. Let us consider a temporal sequence of $3 \mathrm{D}$ images, $\left\{I_{t}\right\}$ where the small rigid displacements have been compensated for, together with the non-linear transformations $T_{t+\delta t \leftarrow t}$.

We start by detecting the temporal extrema of the deformations. We compute the cumulative histogram of the vector modulus $\|\mathbf{v}(m)\|$ (recall that the non-linear transformation is encoded by a vector field) for the membrane points $m \in \mathcal{M}\left(I_{t}\right)$, calculate its $90^{\text {th }}$ percentile, and propose as deformation score $\left(D\left(T_{t+\delta t \leftarrow t}\right)\right)$ the ratio between the mean value of the vector modulus computed on the $10 \%$ membrane points having the largest vector modulus (set denoted by $\mathcal{M}_{10 \uparrow}$ ), and the mean value of the vector modulus computed on the $90 \%$ membrane points having the smallest vector
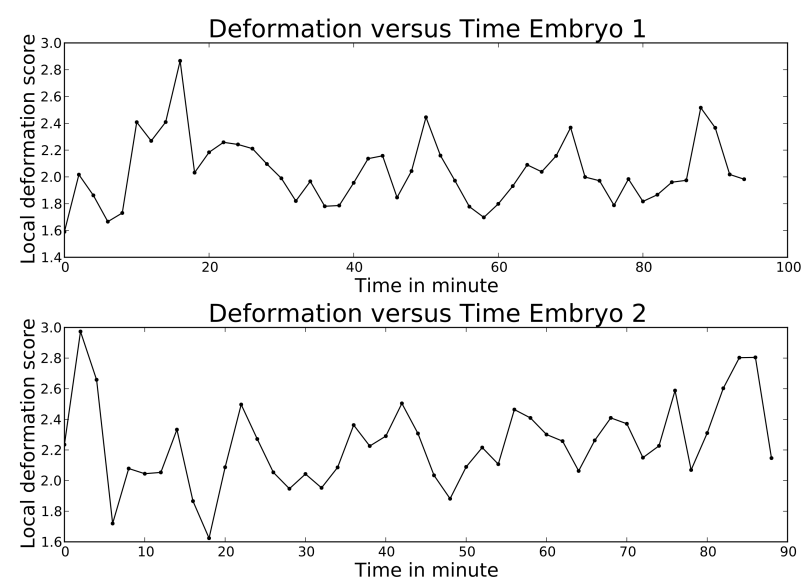

Fig. 4. Score of local deformation over time for the two embryos to be co-registered.

modulus (set denoted by $\mathcal{M}_{90 \downarrow}$ ) :

$$
D\left(T_{t+\delta t \leftarrow t}\right)=\frac{\sum_{m \in \mathcal{M}_{10 \uparrow}\left(I_{t}\right)}\|v(m)\|}{\left|\mathcal{M}_{10 \uparrow}\left(I_{t}\right)\right|}\left(\frac{\sum_{m \in \mathcal{M}_{90 \downarrow}\left(I_{t}\right)}\|v(m)\|}{\left|\mathcal{M}_{90 \downarrow}\left(I_{t}\right)\right|}\right)^{-1}
$$

This deformation score exhibits some extrema with respect to time (Fig. 4), which correspond to the time positions of our 3D+t landmarks, denoted by $t_{i}=t\left(L_{i}\right)$. From now on, we assume that the landmarks are temporally ordered, ie $i<i^{\prime} \Leftrightarrow t_{i}<t_{i^{\prime}}$.

For each of these particular time points, we now compute the connected components of $\mathcal{M}_{10 \uparrow}$, retain the largest of them (this somehow corresponds at extracting the largest, or extremal, deformation), and compute the center of mass of this largest connected component: this yields the spatial positions of our 3D+t landmarks, denoted by $x_{i}=x\left(L_{i}\right)$.

\subsection{Sequences co-registration}

Let us consider two morphogenesis time series $\left\{I_{t}\right\}$ and $\left\{I_{t^{\prime}}^{\prime}\right\}$ and their associated landmarks $\mathcal{L}=\left\{L_{i}\right\}$ and $\mathcal{L}^{\prime}=\left\{L_{j}^{\prime}\right\}$. To register the two time series, we build and test pairing hypothesis between $\mathcal{L}$ and $\mathcal{L}^{\prime}$, this pairing hypothesis is included in the powerset $\mathcal{P}\left(\mathcal{L} \times \mathcal{L}^{\prime}\right)$.

A pairing hypothesis $H=\left\{\left(i_{H}, j_{H}\right)\right\}$ consists of a set of couples representing the landmark pairing $\left\{\left(L_{i_{H}}, L_{j_{H}}\right)\right\}$. Pairing hypothesis are not all tested, and we retain only those obeying: 1) at least 5 landmarks have to be effectively paired; 2) pairings are temporally consistent, ie for two pairings $\left(i_{H}, j_{H}\right)$ and $\left(i_{H}^{\prime}, j_{H}^{\prime}\right)$, if $i_{H}<i_{H}^{\prime}$ then $j_{H}<j_{H}^{\prime}$ (recall that the landmark indexes are temporally ordered). Given a pairing hypothesis, the rigid transformation registering the paired landmarks can be easily estimated in the least squares sense :

$$
T_{H}=\arg \min _{T} \sum_{\left\{\left(i_{H}, j_{H}\right)\right\}}\left\|T\left(x_{i_{H}}\right)-x_{j_{H}}^{\prime}\right\|^{2}
$$

The mean of the residuals measures the hypothesis registration quality, allowing to define the best pairing hypothesis, $\hat{H}$, by

$$
\hat{H}=\arg \min _{H} \frac{1}{|H|} \sum_{\left\{\left(i_{H}, j_{H}\right)\right\}}\left\|T_{H}\left(x_{i_{H}}\right)-x_{j_{H}}^{\prime}\right\|^{2}
$$




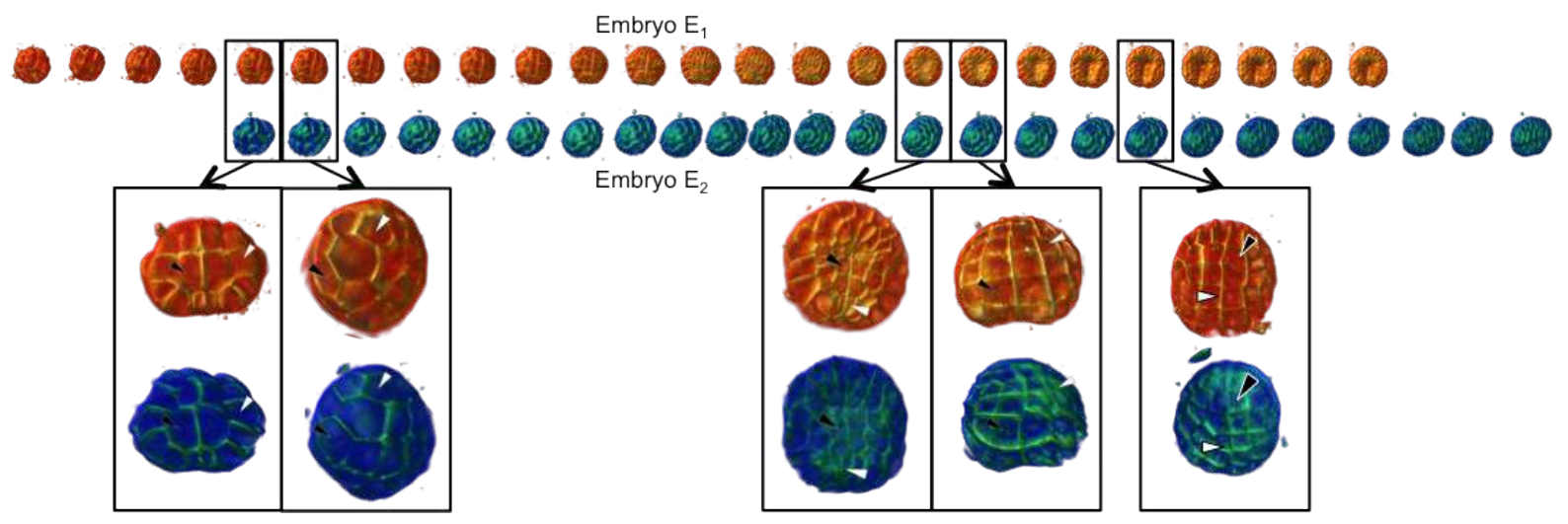

Fig. 5. Spatio-temporal registration of two time-series of embryo. Enlarged renderings indicate the registered times $\left\{\left(t_{i_{\hat{H}}}, t_{j_{\hat{H}}}^{\prime}\right)\right\}$. Notice that the temporal registration is not linear since the interval length between two registered time is different from one embryo to the next.

yielding not only a spatial co-registration of both sequences thanks to $T_{\hat{H}}$ but also a temporal co-registration thanks to the couples $\left\{\left(t_{i_{\hat{H}}}, t_{j_{\hat{H}}}^{\prime}\right)\right\}$. This temporal co-registration is sparse, since only a few time-points are co-registered. A dense temporal co-registration (where all time-points are co-registered) can easily be induced by simple means (e.g. linear interpolation) although more sophisticated schemes are under investigation.

Last, the spatial transformation between the two sequences is refined twice. First, we re-estimate $T_{\hat{H}}$ with a Least Trimmed Squares optimization (which discards the worse pairings out of $\left\{\left(i_{\hat{H}}, j_{\hat{H}}\right)\right\}$ ). Second, we resample the images $I_{j_{\hat{H}}}^{\prime}$ using $T_{\hat{H}}$ and co-register the resulting images $I_{j_{\hat{H}}}^{\prime} \circ T_{\hat{H}}$ with the images $I_{i_{\hat{H}}}$. It yields a number of transformations $T_{\left(I_{j_{\hat{H}}^{\prime}} \circ T_{\hat{H}}\right) \leftarrow I_{i}}$. Averaging them allows to get the spatial transformation $T_{\left\{I_{t^{\prime}}^{\prime}\right\} \leftarrow\left\{I_{t}\right\}}$ that aligns the sequence $\left\{I_{t^{\prime}}^{\prime}\right\}$ onto $\left\{I_{t}\right\}$ (see Fig. 5).

$$
T_{\left\{I_{t^{\prime}}^{\prime}\right\} \leftarrow\left\{I_{t}\right\}}=T_{\hat{H}} \circ\left(\frac{1}{|\hat{H}|} \sum_{\left\{\left(i_{\hat{H}}, j_{\hat{H}}\right)\right\}} T_{\left(I_{j_{\hat{H}}^{\prime}}^{\prime} \circ T_{\hat{H}}\right) \leftarrow I_{i} \hat{H}}\right)
$$

A visual inspection of the registered sequences demonstrates both the temporal re-synchronization of both embryos as well as the superimposition of the corresponding cells (that can be named in the first embryogenesis stages).

\section{DISCUSSION}

We proposed a method to spatially and temporally align two time series of embryos of P.m., with a linear spatial transformation and a non-linear temporal one. It relies on the fact that morphogenesis events, as cellular divisions, induce temporally and spatially localized deformations. The detection of the extremal deformations is used to build sets of 4D landmarks. Assuming that the development of different embryos of the same species is similar, landmarks identified in different embryos can be paired to eventually find the 4 dimension transformation. Our work validates this assumption in the case of the stereotyped embryos of the ascidian P.m. .

By providing a spatio-temporal registration of $3 \mathrm{D}+\mathrm{t}$ time series of images based on membrane deformation, our method opens the way to the geometrical quantification of morphogenesis at a cellular resolution. Finally, from this method we will be able to build longer time series based on fusion of several overlapping smaller time series.

Acknowledgment : We want to thank the Computational Biology Institute and the Fondation pour la Recherche Médicale for partially founding this research

\section{REFERENCES}

[1] E Munro, F Robin, and P Lemaire, "Cellular morphogenesis in ascidians: how to shape a simple tadpole," Curr Opin Genet Dev, vol. 16, no. 4, pp. 399-405, 2006.

[2] J Traas and O Hamant, "From genes to shape: understanding the control of morphogenesis at the shoot meristem in higher plants using systems biology," CR Biol, vol. 332, no. 11, pp. 974-85, 2009.

[3] PJ Keller, "Imaging morphogenesis: technological advances and biological insights," Science, vol. 340, no. 6137, pp. 1234168, 2013.

[4] U Krzic, S Gunther, TE Saunders, SJ Streichan, and L Hufnagel, "Multiview light-sheet microscope for rapid in toto imaging," Nat Methods, vol. 9, no. 7, pp. 730-3, 2012.

[5] R Fernandez, P Das, V Mirabet, E Moscardi, J Traas, JL Verdeil, G Malandain, and C Godin, "Imaging plant growth in 4-d: robust tissue reconstruction and lineaging at cell resolution," Nat Methods, vol. 7, pp. 547-553, 2010.

[6] B Zitová and J Flusser, "Image registration methods: a survey," Image Vis Comput., vol. 21, no. 11, pp. 977-1000, 2003.

[7] A Sotiras, C Davatzikos, and N Paragios, "Deformable medical image registration: a survey," IEEE Trans Med Imaging, vol. 32, no. 7, pp. 1153-90, 2013.

[8] S Ourselin, A Roche, S Prima, and N Ayache, "Block matching: A general framework to improve robustness of rigid registration of medical images," in MICCAI'00. 2000, vol. 1935 of LNCS, pp. 557-566, Springer.

[9] PJ Besl and ND McKay, "A method for registration of 3-D shapes," IEEE Trans Pattern Anal Mach Intell, vol. 14, no. 2, pp. 239-256, 1992.

[10] PJ Rousseeuw and AM Leroy, Robust Regression and Outlier Detection, Wiley \& Sons, New-York, 1987. 